\title{
CARACTERIZACION CLINICO-TERAPEUTICA DE LOS PACIENTES CON TRAUMA PENETRANTE DE CUELLO EN EL HOSPITAL ESCUELA
}

Clinical and therapeutic characterization of patients with penetrating neck trauma in hospital escuela

$$
\text { C.E.R }{ }^{1} \text { Dr. Víctor Quiñónez Altamirano }{ }^{2} \text { Dr. Francisco Ayes Valladares }{ }^{3}
$$

1 Comité Editorial de la Revista de Postgrados de Medicina UNAH, Dirección de Docencia e Investigación-HE: Dr. Carlos Vargas Pineda, Dr. Rolando-Aguilera-L, Dra. Guadalupe-Romero-A, ${ }^{2}$ Médico residente III año Cirugía general. UNAH. ${ }^{3}$ Cirujano torácico general. Catedrático titular III. UNAH

RESUMEN. OBJETIVO. Establecer el comportamiento clínico y la terapéutica de las lesiones (aerodigestivas, vasculares, neurológicas, y de otra índole) en el trauma penetrante de cuello.

PACIENTES Y METODOS. Estudio prospectivo descriptivo que involucro a los pacientes que ingresaron a las salas de cirugía del Hospital Escuela entre el 1ro de febrero del 2008 y el 30 de agosto del 2010, con diagnostico de trauma penetrante de cuello. Se aplico un instrumento de recoleccion de datos clínicos y de manejo del paciente. Los resultados se presentan como frecuencias $y$ porcentajes de las variables estudiadas utilizando el sistema de proceso de datos EPI.INFO 3.5.1

RESULTADOS. $97.30 \%$ de los pacientes con trauma penetrante de cuello son masculinos. La edad media encontrada es de 36.6 años. $51.40 \%$ de los traumas fue producido por arma blanca. La zona II de
Monson fue lesionada en un 86.50\%.la mayoría de los pacientes provienen de Francisco Morazán 73\% la mayoría de los pacientes sufrió de lesiones de la vía aérea $32.40 \%$. $21.6 \%$ de los pacientes no tuvieron ningún tipo de lesión. El procedimiento más efectuado fue la cervicotomía izquierda en $48 \%$.un paciente murió a consecuencia de neumonía nosocomial, además de presentar de lesión de la vía aérea, también como factores de comorbilidad tenía su edad 92 años, dpc. Las cervicotomias no terapéuticas fueron 8 (21.6\%)

CONCLUSION. Los traumas penetrantes de cuello atendidas en este centro se asociaron a lesiones de vía aérea en $32.40 \%$ y vasculares $18.90 \%$ de los casos. La cervicotomia fue el manejo de elección para todos los casos encontrando muy pocos casos de complicaciones $18 \%$ y muerte $1 \%$ 
Palabras claves: zona de Monson, lesión aero-digestiva, cervicotomia, DPC (desnutrición proteica calórica

ABSTRACT.OBJECTIVE. to establish the clinical behavior and treatment of injuries (aero-digestive, vascular, neurological or else) in penetrating neck trauma.

\section{PATIENTS AND METHODS.}

Descriptive study involving patients admitted to the surgical room of Hospital escuela from February 2008 through august 2010 whit diagnosis of penetrating neck trauma. We applied a data collection instrument for clinical findings and patient management. The results are presented as frequencies and percentages of study variables using the data processing system EPI.INFO 3.5.1

RESULTS. $97.30 \%$ of patients with penetrating neck injuries were male. The average age was $36.6 \%$. $51.40 \%$ of injuries were caused by cool steel. Monson zone II was injured in $86.50 \%$. The majority of patients come from Francisco Morazán 73\%. The majority of patients suffered injuries in the air way $32.40 \%$. $21.6 \%$ of the patients did not have any type of injury. The procedure more made was the cervicotomía left at $48 \%$. A patient died as a result of nosocomial pneumonia, in addition to submit of injury of the air way, also as factors of morbidity had its age 92 years, dpc. The cervicotomias no therapeutic were $8(21.6 \%)$.
CONCLUSION. The penetrating traumas of neck met in this center will be joined by injuries to air in $32.40 \%$ and vascular $18.90 \%$ of the cases. The cervicotomia was the handling of choice for all the cases finding very few cases of complications 18 per cent and death $1 \%$.

INTRODUCCION. Los traumas penetrantes de cuello que se presentan en el Hospital escuela son una entidad cuya frecuencia está en constante ascenso. en vista que en los últimos años este tipo de trauma ha sufrido un ascenso acorde al índice de violencia que vive nuestro país, y debido a la mortalidad elevada que implica las lesiones de cuello nos vemos en la obligación de investigar a fondo el manejo optimo de dichas lesiones para así dar mayor calidad de atención y disminuir el riesgo de complicaciones y muerte en estos pacientes victimas de trauma de cuello. Esta pequeña zona concentra una cantidad de estructuras vitales que lesionadas pueden poner en riesgo la vida del paciente en forma inmediata, por lo que su correcto manejo exige un conocimiento profundo de la anatomía de la región y son un desafío aun para los cirujanos más experimentados. El manejo de estas heridas ha sufrido amplias variaciones a lo largo del tiempo, desde un manejo quirúrgico mandatorio de todos los pacientes, hasta un manejo selectivo, basado en 
los hallazgos clínicos y los estudios diagnósticos donde el advenimiento de nuevas técnicas de imágenes han permitido el desarrollo de procedimientos diagnósticos y terapéuticos de fundamental importancia en estas lesiones. Estos cambios en el manejo han acompañado en el tiempo a los cambios en los mecanismos lesiónales, donde las heridas de guerra con proyectiles de gran calibre $\mathrm{y}$ alta velocidad han dado paso a las lesiones de la vida civil, donde la violencia social es la principal causa etiológica de estas heridas en la actualidad.

Las lesiones aero-digestivas y vasculares en particular se asocian en porcentaje variable a pocas o ninguna manifestación clínica y esto hace; si la evaluación se basa únicamente en la clínica, que muchas lesiones pasen desapercibidas y sean manejadas cuando sus complicaciones se hacen evidentes, aumentando así, no solo la morbilidad sino también magnitud de las secuelas y la mortalidad.

Las nuevas modalidades diagnosticas y el tratamiento adecuado y oportuno basado en protocolos de acción y no en conductas empíricas de las lesiones aero-digestivas, vasculares y neurológicas del cuello han desembocado en una significativa disminución de la morbi-mortalidad y por ende de los gastos que se deducen por la atención de estos pacientes en el sistema de salud.
PACIENTES Y METODOS. Metodología: Estudio prospectivo descriptivo; que involucro a los pacientes que ingresaron a las salas de cirugía entre el 1ro de febrero del 2008 y el 30 de agosto del 2010 con diagnostico de trauma penetrante de cuello. En vista de que la cantidad total de casos era de 37 se tomo como muestra el universo para que fuera representativa la muestra. Se recopilo un total de 37 traumatismos penetrantes de cuello. Se aplicaron las siguientes variables: nombre, expediente clínico, sexo, edad, mecanismo de lesión, procedencia, tipo de lesión, área anatomica afectada, cirugía realizada, complicaciones.la información se recopilo manualmente. Se calculo frecuencias, medianas y porcentaje de las principales variables a través del programa EPI. Info versión 3.5.1 2000.

\section{RESULTADOS.}

Treinta y seis casos (97.30\%) correspondieron al sexo masculino.la edad de los pacientes oscilo entre los 18 y 92 años con una media de 36.6 años. Con respecto al mecanismo de lesión 19(51.4\%) de los casos se debió a herida por arma blanca y 18(48.6\%) se debió a herida por proyectil disparado por arma de fuego. Las zonas de cuello lesionadas se describen en el cuadro $\mathrm{N}^{\circ} .1$ 
Cuadro $\mathrm{N}^{\circ} .1$

zona anatomica afectada en los pacientes con trauma penetrante de cuello

\begin{tabular}{|l|r|r|}
\cline { 2 - 3 } \multicolumn{1}{c|}{ Zona del cuello* } & Con Lesión & Sin lesión \\
\hline Zona I & 8 & 29 \\
\hline Zona II & 32 & 5 \\
\hline Zona III & 3 & 34 \\
\hline
\end{tabular}

*zonas de Monson

Las zonas de procedencia $q$ reportaron más casos en este centro asistencial fue francisco Morazán con 27(73\%); luego la paz 3(8.10\%); el paraíso $2(5.40 \%)$; valle $2(5.40 \%)$; santa barbará 1(2.70\%); Comayagua $1(2.70 \%)$

Los rangos de edad se dividieron encontrando que el trauma de cuello fue más frecuente en pacientes de 18-33 años 19(51\%); luego 34-50 años 12(32\%); con menos frecuencia los pacientes de la tercera edad mayores de 65 años 4 (11\%); 51-64 años 2 (5\%).

Las lesiones encontradas más frecuentemente en estos pacientes fueron: Lesiones de vía aérea 12(32.40\%); lesiones vasculares $7(18.90 \%)$; lesiones de faringe 5(13.50\%); lesiones de esófago 4(10.80\%); lesión de tiroides $3(8.10 \%)$; y un gran porcentaje de pacientes no tuvieron lesión al momento de ser intervenidos
$8(21.60 \%)$. El manejo que se le dio a estas lesiones siempre fue la cervicotomía como método de exploración estándar, el manejo se dio según los hallazgos transoperatorios, por lo que se darán en número ya que se practico más de un procedimiento en un mismo paciente: cervicotomía izquierda (17); cervicotomía derecha (10); drenaje (10); cierre primario (9); ligadura vascular (6); cierre primario mas parche muscular (2); traqueostomía. Grafico N¹:

\section{GRAFICO $N^{\circ} .1$}

\section{Manejo quirúrgico realizado en pacientes con trauma de cuello}

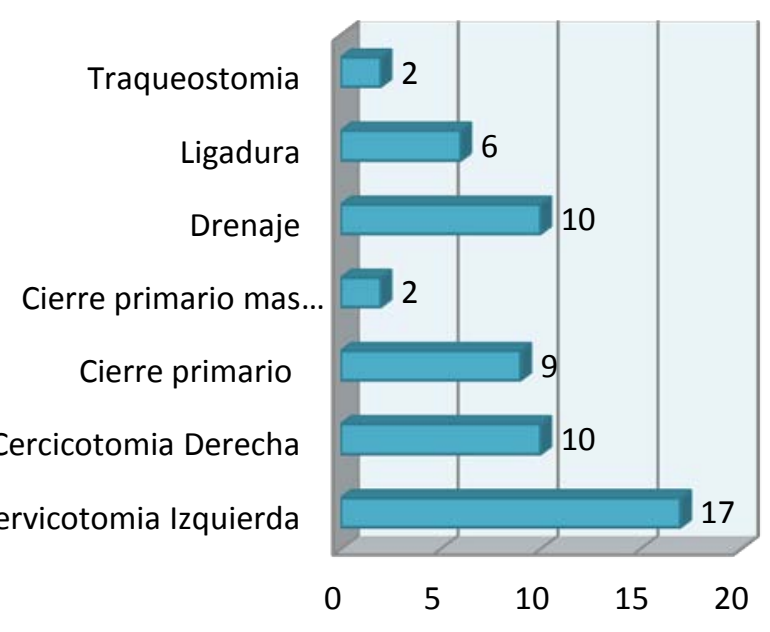


En su gran mayoría estos pacientes no experimentaron complicación alguna, evolucionando satisfactoriamente y egresando sin dificultades 30 (81.10\%) sin lesiones; el resto de complicaciones se presentaron solo $1(2.70 \%)$ vez; dehiscencia de sutura, dehiscencia sutura tráquea, disfonía, enfisema, fuga de rafia de esófago, neumonía, muerte, paraplejía.

\section{DISCUSION.}

El trauma de cuello constituye el 5 al $10 \%$ de los traumatismos. El abordaje inicial y el manejo de los pacientes con traumas penetrantes de cuello ha presentado diversas etapas en el transcurso del tiempo. Existe el periodo de exploración mandatoria que evoluciono mas tarde a la exploración selectiva con protocolización sistemática de estos pacientes, hasta las conductas meramente empíricas que basan el manejo en la ausencia o presencia de manifestaciones externas de lesión. Los problemas que genera abarcan la muerte súbita y las complicaciones en el tratamiento conservador quirúrgico. Coincidimos con la literatura revisada en que las personas menores de 45 años involucran la gran mayoría de los casos. (1) en este estudio el $96.3 \%$ de los pacientes pertenece al sexo masculino. El mecanismo de lesión más frecuente fue el de herida por arma blanca superando por muy poco a las lesiones por proyectil disparado por arma de fuego que en la literatura son la causa más frecuente de trauma penetrantes de cuello. (1-3). La zona II del cuello involucra el $86.5 \%$ acorde con los reportes nacionales e internacionales (4-7). El manejo de los pacientes sin evidencia clínica de lesiones especifica y con estabilidad hemodinámica, se basa en los estudios diagnósticos realizados de acuerdo a la zona del cuello afectada en aras de evitar cirugías innecesarias o diferir las que lo son por juicios empíricos. Todo de acuerdo a los lineamientos de la sociedad americana para la cirugía de trauma. $(2,5)$. En este estudio se reportaron 8 exploraciones no terapéuticas.

En el manejo de las lesiones aerodigestivas se prefirió el cierre primario y en casos extremos la resección traqueal con anastomosis sin traqueostomía y parche muscular en caso de lesiones traqueo-esofágicas. Todo en concordancia con la literatura internacional. (1,6-9,14-17) las lesiones venosas se manejaron con ligadura y se intento la reparación de la carótida antes de su ligadura.las complicaciones dependen del tiempo que transcurre entre el trauma y la intervención.

\section{CONCLUSIONES.}

El trauma penetrante de cuello sigue siendo poco frecuente pero su complejidad y alto índice de complicaciones y mortalidad si no son manejadas adecuadamente. Los traumas penetrantes de cuello 
atendidos en nuestro centro presentan lesiones aero-digestivas en el $42 \%$ y vasculares en $18 \%$ de los casos.

Es importante remarcar que cualquiera que sea el protocolo que se utilice, resulta fundamental descartar la lesión de esófago cervical, porque la mortalidad cuando esta se repara de inmediato es de $2 \%$; cuando se pasa inadvertida y se realiza tardíamente el cierre la mortalidad es de $44 \%$ y aumenta cuanto más se demore la intervención, llegando al 100\% cuando no se realiza. Un gran porcentaje de casos ingresan con lesiones de este tipo pero sin el hallazgo clínico que las sugieran. Por tanto, la aplicación de protocolos es la única forma de señalar la conducta adecuada y sigue estando por encima de la experiencia empírica.

\section{RECOMENDACIONES:}

En vista de el grado de dificultad del manejo de la lesiones de cuello se recomienda que el manejo de estas sea por un cirujano capacitado y con experiencia en el manejo de las mismas.

Ante los distintos cambios que se ha dado sobre el manejo del trauma de cuello a través del paso del tiempo y según la literatura consultada el manejo de estas lesiones debe individualizarse en cada paciente y debe hacerse uso correcto de los actuales métodos diagnósticos con el fin de identificar tempranamente lesiones que comprometan la vida y pongan en inminente peligro al paciente.

Se debe lograr identificar tempranamente los datos que indiquen un manejo quirúrgico temprano o un manejo conservador de inicio, siendo objetivos en que la evolución de las lesiones de cuello es muy dinámica y su manejo puede cambiar de un momento a otro según sea su evolución clínica

\section{BIBLIOGRAFIA.}

1. Peralta, R; Hurford, W. Airway Trauma. Int Anesthesiol Clin 2000;

38(3):111-27

2. Britt, L; Peyser, M. Penetrating and Blunt Neck Trauma, en Moore, EE;

Mattox, KL; Feliciano, DV; (eds): Trauma, Ed 4.

Mc Graw-Hill, New York

2000, pp 437-50

3. Fogelman, MJ; Stewart, RD. Penetrating wounds of the neck. Am J Surg

1956; 91:581

4. Ríos Bruno, G; Castiglioni, JC; Bergalli, L; Gateño, N; Torterolo, E;

Crespo, LE; et al. Heridas de cuello. Cir Urug 1974; 44(4):225-66

5. Ayuyao, AM; Kaldezi, YL; Parsa, MH.

Penetrating neck wounds.

Mandatory versus selective exploration. Ann Surg 1985; 202:563

6. Roth, B; Demetriades, D. Penetrating trauma to the neck. Current Opinion

In Critical Care 1999; 5(6):482-7

7. Jurkovich, G. Trauma: Definitive Care Phase: Neck Injuries, en Greenfield,

LJ; Mulholland, M; Oldham, KT; Zelenock, GB; Lillemoe, KD; (Eds):

Surgery: Scientific Principles and Practice. Ed 3. Lippincott

Williams\&Wilkins, Philadelphia 1997, pp 309-17

8. Puig, R; Boudrandi, S; Bado, A. Heridas de

cuello. Análisis de cinco

Observaciones y breve revisión para una propuesta de normatización. Cir Urug 1989; 59:15-23

30

9. Laviña, R; Misa, C; Silva, C. Anatomía del cuello, en Silva, C; Laviña, R;

Misa, C; (eds): Cuadernos de Semiología y Clínica Quirúrgica. Cuello. Ed 1.

Librería médica s.r.l., Montevideo, pp 5-41 


\section{Revista de los Postgrados de Medicina UNAH \\ Vol. $13 \mathrm{~N}^{\circ} 3$ Agosto-Octubre 2010}

10. Shatz, D; Kirton, O; McKenney, M; Civetta, J.

Penetrating neck injuries,

En Shatz, D; Kirton, O; McKenney, M; Civetta;

(Eds): Manual of Trauma and

Emergency Surgery. Ed 1. W. B. Saunders

Company, Philadelphia 2000, pp

34-53

11. Saletta, JD; Lowe, RJ; Leonardo, TL.

Penetrating trauma of the neck. J

Trauma 1976; 16:579

12. Ventura, G. Traumatismos de cuello. Emerg

Uruguay 1985; 5(3):166-9

13. Ordog, GJ; Albin, D; Wasserberger, J. Shotgun

"bird-shot" wounds to the

Neck. J Trauma 1987; 28:491

14. Demetriades, D; Asensio, J; Velmahos, G;

Thal, E. Complex problems in

Penetrating neck trauma. Surg Clin North Am

1996; 76:661-81

15. Pate, JW. Tracheobronchial and esophageal

injuries. Surg Clin North Am

1989; 69:111-23

16. Ordog, GJ. Penetrating neck trauma. J Trauma $1987 ; 27: 483$

17. Jurkovich, GJ; Zingerelli, W; Wallace, J;

Curreri, PW. Penetrating neck

Trauma: Diagnostic studies in the asymptomatic

patient. J Trauma 1985; 25:819 